II. Aus der Abtheilung für Ohrenkranke des Charitékrankenhauses in Berlin.

(Dirigirender Arzt: Prof. Dr. Trautmann.)

\section{Zur operativen Behandlung der otitischen Hirnhautentzündungen.}

\section{Von Stabsarzt Dr. Richard Müller.}

Die im letzten Jahrzehnt ausgebaute Lehre von der chirurgischen Behandlung der Mittelohreiterungen hat es mit sich gebracht, dass die Ohrenärzte auch die vom $\mathrm{Ohr}$ ausgehenden Erkrankungen der Nachbarorgane in den Bereich ihrer operativen Thätigkeit gezogen haben. Das gilt besonders von den intracraniellen Krankheiten otitischen Ursprungs, und die Zuständigkeit des Ohrenarztes auf diesem Gebiete wird heute wohl von niemand mehr bezweifelt. Die Hirnhäute, die Hirnblutleiter und das Gehirn selbst können im ursüchlichen Zusammenhange mit einer Mittelohreiterung erkranken, und jedes dieser Organe kann daher Gegenstand eines ohrenärztlichen operativen Eingriffs werden. Am Gehirn und an den Hirnblutleitern ist das bisher häufiger der Fall gewesen als an den Hirnhäuten, doch sind auch die Krankheiten dieser letzteren einer operativen Behandlung nicht unzugängig. So ist es bekannt, dass eine beginnende eiterige Meningitis durch ergiebige Freilegung der erkrankten Partie und nachfolgende kräftige Drainage mittels der aufgelegten Verbandstïcke noch "coupirt" und damit geheilt werden kann, wenn vorher die zu Grunde liegende Mittelohreiterung operativ geheilt, bezw. ihre Heilung durch die Operation eingeleitet worden ist.

In neuerer Zeit nun haben Quincke, ${ }^{1}$ v. Bergmann, ${ }^{2}$ ) Boenninghalls ${ }^{3}$ ) und andere die Aufmerksamkeit auf die seröse Form der Meningitis gelenkt. Dass auch diese Form, ebenso wie die eiterige, im Anschluss an Mittelohrentzündungen oder deren Folgezustände vorkommt, kann nach den Ausführungen der genannten Autoren nicht mehr zweifelhaft sein und ebensowenig, dass dieses Leiden einer operativen Behandlung zugängig ist. Die zwei Fälle, welche ich im Folgenden bekannt geben will, sind geeignet, diese Behauptung zu stützen.

Beide Fälle haben das Gemeinsame, dass in beiden - allerdings mit dem nöthigen Vorbehalt - die Diagnose auf einen otitischen Gehirnabscess gestellt worden war, dass aber bei der Operation kein Abscess gefunden wurde. Und doch kam es in beiden Fällen nach der Operation zur Heilung, gerade durch den operativen Eingriff, nicht trotz desselben. Den Entschluss, zu operiren, hatten wir in beiden Fällen trotz der Unsicherheit der Diagnose hauptsächlich mit Rücksicht darauf gefasst, dass in einer Operation die einzige Möglichkeit einer Rettung lag, während ohne operatives Handeln der tödtliche Ausgang unvermeidlich war. Der Verlauf hat gelehrt, dass bei der einen Patientin eine Meningitis serosa externa chronica, bei der anderen eine Meningitis serosa ventricularis s. interna acuta vorlag.

\section{a. Meningitis serosa externa chronica.}

Auguste K., 23 Jahre. Anfang April 1895 acute Mittelohreiterung links nach Influenza. 26. April 1895 Aufnahme auf unsere Klinik. Fieber, Schwellung der Weichtheile um das linke Ohr, Processus mastoideus druckempfindlich, Gehörgang schlitzförmig verengt. Flüistersprache 1. nur dicht am Ohre gehört. 1. Mai Aufmeisselung des Antrum mastoideum (nicht Radicaloperation): Empyem des Warzenfortsatzes; nach hinten oben wird die Dura in doppelter Linsengrösse freigelegt. Heilung erst um Weihnachten 1895 vollendet. Körpergewicht damals 120 Pfund.

In der ganzen Zeit von 1896-1898 will sie beständig an mässigen Kopfschmerzen und Schwindel und zeitweise auch an Schmerzen im linken Ohr gelitten haben; ihre Arbeit als Dienstmädchen konnte sie trotzdem verrichten. Anfang September 1898 aber wurden plötzlich ohne bekannte Ursache die Beschwerden ärger, am 9. September Erbrechen, am 10. September 1898 Aufnahme in die Charité.

Klagen: Heftige, linksseitige Kopfschmerzen; Schwindel, so dass sie nicht allein gehen kann, sondern geführt werden muss; grosse Schwäche; Schlaf- und Appetitlosigkeit, Brechneigung; Benommenheit.

Status: Mittelkräftiges, etwas blutarmes Mädchen mit gesmunden inneren Organen; erblich nicht tuberkulös belastet; keine Anzeichen

1) Ueber Meningitis serosa. Sammlung klinischer Vorträge, neue Folge, innere Medicin, No. 23.

$\left.{ }^{2}\right)$ Die chirurgische Behandlung von Hirnkrankheiten. 3. Auflage. 1899. S. 149

3) Die Meningitis serosa acuta. Wiesbaden, J. F. Bergmann, 1897. von Tuberkulose oder Syphilis. Harn frei von Zucker und Eiweiss. Körpergewicht 104 Pfund. Das Mädchen macht einen schwerkranken Eindruck. Bei intensiverem Befragen giebt sie zwar richtige Antworten, ist aber sonst stark benommen und fällt leicht ab. - Schädel auf der ganzen linken Seite stark percussionsempfindlich, besonders über dem Ohr, rechts nicht. Kribbeln und Taubheitsgefühl im rechten Arm, öfter Gefülll des Einschlafens im rechten Bein; Sensibilität der ganzen rechten Seite für blosse Berührung und für sclmer\%hafte Nadelstiche deutlich herabgesetzt. Reflexe normal. Keine Lähmung, aber deutliche Schwäche der Muskeln der ganzen rechten Seite. Leichte Ptosis des linken oberen Augenlids. Facialis in Ordnung. Romberg positiv, fällt dabei nach links trotz der Schwäche der Muskulatur rechts; Gang ganz unsicher, stark schwankend; wenn sie nicht geführt wird, fällt sie alsbald nach links. -- Pupillen mittelweit, reagiren; starke venöse Hyperämie der Netzhaut und der Papillen beiderseits, aber keine deutliche Neuritis. - Keine Nackensteifigkeit, keine Aphasie. Puls in grösseren Zwischenräumen wechselnd, aber in sich nicht unregelmässig, im allgemeinen verlangsamt, um 60 , sehr voll, kräftig. $A$ bendtemperaturen um $37,6^{\circ}$. Ohren: WL,$+ R i R+L-$ Perceptionsdauer stark abgeküızt, wohl in Folge der Benommenheit. - Rechts geringe Retraction, sonst normal; Flüstersprache bei gespannter Aufmerksamkeit normal. Links mässige Retraction, verwaschener Reflex. Keine Eiterung. Flüstersprache $1 \mathrm{~m}$. Hinter dem linken $\mathrm{Ohr}$ strichförmige Narbe von der alten Antrumoperation, Weichtheile dort äusserlich normal, nicht infiltrirt, aber Druck auf die Narbe sehr schmerzhaft, selbst bei Druck durch den hydropathischen Umschlag hindurch zuckt sie zusammen. - Rasche Verschlechterung des Zustandes, zunehmende Somnolenz, öfter Erbrechen. Terstopfung. - Am 15. September zeitweise Bewusstlosigkeit, stöhnt viel vor sich hin. Athmung tief, langsam. Puls 60, sehr voll und kräftig. Dass das Erbrechen besonders in einer bestimmten Seitenlage aufträte, wird nicht bemerkt.

Eine sichere Diagnose war nicht zu stellen; doch sprach die Schmerzhaftigkeit am Processus mastoideıs für entzündliche Vorgänge in diesem, und der sonstige Zustand für eine von dort ausgehende cerebrale Complication - möglicher Weise Schläfenlappenabscess, namentliclı mit Rücksicht auf die rechtssseitigen Sensibilitäts- und Motilitätsstörungen.

Ich bescliloss daher - mein Chef war abwesend -, unter allen Umständen zunächst die alte Antrumoperationswundhöhle wieder freizulegen und je nach dem Befunde weitere Maassnahmen zu ergreifen. Das führte mich am 15. September 1898 zur Radicaloperation links und im Anschluss daran zur Trepanation anf den linken Scliläfenlappen. Dauer $1 \frac{1}{2}$ Stunde.

Nach Durchschneidung der Haut genau in der alten Antrumoperationsnarbe bot sich ein ganz überraschender Befund. Man gelangte sofort in eine grosse, der alten Antrumoperation entsprechende Knochenhöllle ohne jeden Inhalt, weder Bindegewe be noch auch Granulationen fanden sich vor. Die ehemalige Füllung der Wundhöhle musste also wieder resorbirt worden sein. Die Wände der Höhle waren absolut trocken, wie an einem macerirten Schädel, und sahen schwarz, nekrotisch aus. Unter dieser gänzlich nekrotischen Schicht fanden sich zahlreiche, zum Theil recht grosse Zellen, die alle ebenfalls ohne Inhalt und vollständig trocken waren, ihre Wandungen waren grauweiss und machten auch einen abgestorbenen Eindruck wie an dem Schädel eines Skeletts. Es musste nach allen Richtıngen hin ziemlich viel von diesem nekrotischen Knochen weggenommen werden, ehe der Knochen anfing, feucht zu werden und normales Aussehen darzubieten; eine scharfe Demarkationslinie gab es nicht. Der Sinus war weit zurïckgelagert und wurde nicht freigelegt. Die Dura war in der früher freigelegten Ausdehnung verdickt, missfarbig grauschwarz und ausserlich ebenfalls vollständig trocken; sie wird ringsherum bis zur Grösse eines Pfennigstiicks weiter freigelegt, die neu blossgelesten Partieen an der Peripherie nähern sich dem normalen Aussehen. Hintere Wand des knöchernen Gehörgangs vollständig ausgetrocknet, oberes und unteres Blatt der oberen Gehöroangswand ziemlich weit von einander entfernt, zwischen ihnen trockene Zellen. Hammer und Ambos gesund. Horizontaler Bogengang springt stark vor, glänzend weiss, gesund. Uebliche Lappenbildung

Mehrmalige Probepunction durch die freigelegte Dura in den Schläfenlappen; kein Eiter. Beim Znr ückziehen aspirirt die Nadel jedesmal, wenn die Spitze den Raum zwischen Dura und Araclmoidea, bezw. Arachnoidea und Pia passirt, mit la u tem Schlürfen etwas seröse Flüssigkeit. Incision der freigelegten Dura, Eindringen mit dem Messer $3 \mathrm{~cm}$ weit nach oben; kein Eiter, auch als das Messer rechtwinkelig gegen die erste Schnittrichtung auf die Kante gestellt wird. Von einer grösseren Erweiterung des Schnittes mit der Kornzange wird mit Rücksicht auf den möglichen Hirnprolaps in die Wundhöhle hinein Abstand genommen.

Da erfahrungsgemäss eine Probepunction und selbst Probeincision auch bei vorhandenem $A$ bscess negativ ausfallen kann, wurde zur 'Tre panation auf den Schläfenlappen von aussen her geschritten. Verlängerung des ersten Hautschnitts wagerecht nach vorn $6 \mathrm{~cm}$ weit. Ausmeisselung eines Knochenrechtecks aus der Schuppe, $3 \mathrm{~cm}$ lang, reichlich $1 \mathrm{~cm}$ hoch, die untere Lüngskante $1 \mathrm{~cm}$ ïber dem höchsten Punkte des Porus acusticus externus, halb vor, halb hinter der durch diesen 
Punkt gezogenen Senkrechten gelegen. Dura von normaler Farbe, pulsirt nicht. Sie wird zunächst allein in der Längsrichtung des Knochenrechtecks von vorn nach hinten durchschnitten; als bald entleert sich seröse Flüssigkeit in reichlicher Menge und anscheinend unter ziemlich hohem Drucke, derart, dass das von aussen her über die Dura fliessende Blut durch die seröse Flüssigkeit fortgesetzt kräftig bei Seite geschwemmt wird. Nun wird mit dem Messer nach allen Richtungen hin in den Schläfenlappen auf etwa $2 \frac{1}{2}-3 \mathrm{~cm}$ eingegangen; kein Eiter, auch dann nicht, als die Schnittwunde im Schläfenlappen mit der Kornzange erweitert wurde. Einführung eines $6 \mathrm{~cm}$ langen, $2 \mathrm{~cm}$ breiten drainirenden Jodoformgazestreifens in die Hirnwunde. Radicaloperationshöhle mit Jodoformgaze austamponirt. Puls am Schluss der Operation 60, voll, kräftig.

Nachmittags 5 Uhr: $37,0^{\circ}$. Puls 84 . Sensorium frei. Der sehr grosse und dicke Verband ist hinten durchfeuchtet; Auflegen neuer Compressen über den alten Verband in reichlicher Menge.

16. September. Kein Kopfschmerz; fühlt sich iuberhaupt wohler. Verband auch durch die neu aufgelegten Compressen hindurch gänzlich durchtränkt, wie wenn man den Kopf mitsammt dem Verbande in einen Eimer Wasser gesteckt hätte. Daher Verbandwechsel bis auf die Tampons in der Radicaloperationshöhle, welche liegen bleiben. Keine Blutung, kein Blutgerinnsel zwischen Wunde und innerster Verbandschicht. $36,9^{\circ} .81$.

17. September. Sensorium ganz frei. 37,0. 84. Händedruck beiderseits gleich stark, keine Schwäche auf der ganzen rechten Seite mehr zu bemerken. Subjectives Befinden nach spontaner Aussage der Kranken gut, kein Kopfschmerz. Keine Spur von Ptosis mehr. Verband wieder durch und durch zum Auswinden serös durchtränkt, einschliesslich der Binde; Verbandwechsel, Auflegen von Verbandstücken in grosser Menge. Kein Erbrechen mehr, fängt an, feste Speisen zu sich zu nehmen.

19. September. Verband von vorgestern wieder stark durchtränkt, doch sind heute früh die äusseren Partieen wieder eingetrocknet, was auf ein Nachlassen der Secretion schliessen lïsst. Verbandwechsel.

21. September. Verbandwechsel; Verbandstïcke immer noch stark durchtränkt, aber nicht mehr zum Auswinden. Die Verbandstücke werden mit etwas Wasser ausgelaugt; die Trommer'sche Probe fällt mit dieser Flüssigkeit negativ aus. - Erster Verbandwechsel an der Radicaloperationswınde: Wundhöhle sieht correkt aus.

23. September. Verbandwechsel. Wundsecretion geringer. Kein Hirnprolaps. Wohlbefinden. Appetit gut.

29. September. Heute Secretion wieder etwas stärker, womit die vermehrten Kopfschmerzen der letzten zwei Tage erklärt sein dürften. Stuhl spontan. Sitzt aufrecht im Bett; während des Aufrichtens etwas Schwindel. - Augenhintergrund normal, keine Hyperämie mehr. Puls in letzter Zeit zwischen 70 und 80 , Temperatur um $37,0^{\circ}$, einige Male Abends $37,4-37,6^{\circ}$.

Der weitere Verlauf gestaltete sich mit geringen, nicht nennenswerthen Zwischenfällen vollständig normal. Am 14. October wurden noch $3-4 \mathrm{~cm}$ Gaze in die Hirnwunde eingeführt, vom 22 . ab wurde sie nicht mehr drainirt; 17 . November Hirnwunde geheilt. Die Kranke ist den grössten Theil des Tages ausser Bett, kann aber, trotzdem sie sich in den Beinen kräftig fühlt, noch nicht gehen, weil sie schwindelig ist und beim Gehen nach links hinüberfällt. Druck auf die Schläf ennarbe löst Schwindelgefühl aus. Sonst keine Beschwerden.

24. November. Transplantation zweier Hautläppchen vom Vorderarm in die Radicaloperationswunde. - 17. Januar 1899. Körpergewicht von 104 auf 114 Pfund gestiegen. Temperatur schon seit Wochen Abends öfter bis $37,8^{\circ}$.

21. Januar 1899. Radicaloperationswunde geheilt. Fliistersprache $1.1 \mathrm{~m}$. Die Kranke kann noch immer nicht ohne Stütze gehen; im Gehstuhl kann sie sich ganz gut fortbewegen; auch kommt sie, wenn sie sich an Gegenständen fortgreifen kann, ziemlich schnell vorwärts. Macht jetzt Gehübungen mit einem Stocke. - Zeitweise wieder geringe Kopfschmerzen.

15. Mär. Gehvermögen etwas gebessert, kann aber immer noch nicht allein gehen; Schwindel, Fallen nach links. Augenhintergrund normal. Herabsetzung der Sensibilität auf der ganzen rechten Körperhälfte, besonders hochgradige Hypalgesie rechts. - $\mathrm{WL}+, \mathrm{Ri} R+, \mathrm{L}-$; Perzeptionsdauer nicht abgekürrzt. Flüstersprachel. für Zahlen $5 \mathrm{~m}$. Durch Luft wird die gesammte Tonreihe links bis hinunter zu 60 v. d. gehört, darunter nicht mehr. Gehirn- und Ohrwunde fest verheilt. Mit Riicksicht auf die Kleinheit des Knochendefects in der Schläfenschuppe und auf die Festigkeit der Narbe, die absolut unempfindlich ist - auch kein Schwindelgefühl bei Druck darauf - , braucht kein Schutzverband getragen zu werden.

10. April. Da in dem Zustande seit Wochen keine Aenderung: mehr eingetreten ist, wurde für die Kranke die Aufnahme in ein Siechenhaus beantragt. ${ }^{1}$ )

1) Leider hat sich seitdem wieder eine Verschlimmerung eingestellt: Kopfschmerz, Percussionsempfindlichkeit der linken Schädelhälfte, öfter Erbrechen. Gewichtsabnahme. (Augenhintergrund normal, Puls nicht verlangsamt, kein Fieber). Von einer Entlassung musste daher einstweilen Abstand genommen werden.
Von einer vollständigen Heilung der Krranken kann mit Rücksicht auf das noch bestelıende Schwindelgefühl, das mangelhafte Gehvermögen, die Herabsetzung der Sensibilität der rechten Seite und die zeitweise in letzter Zeit wieder beobachteten Kopfschmerzen --- sowie auf die jüngst aufgetretene Verschlimmerung (siehe Fussnote) - niclit die Rede sein; doch hat die Operation am 15. September 1898 zweifelsolme durch Beseitigung der monıentan drohenden Jebensgefahr unmittelbar lebensrettend gewirkt, und es ist auch zu einer endgiltigen Heilung der. Wunden gekommell.

Zur Diagnose im vorliegenden Falle sei folgendes bemerkt: Die symptome, welche die Kranke vor der Operation darbot, wiesen, namentlich auch mit Rücksiclit auf das Fehlen krankliafter Ersclicinungen an anderen Organen, mit Bestimmtlıeit darauf hin, dass eine schwere, das Leben bedrohende Affection des Gehirns vorlag, und die gleichzeitig bestehende Druckempfindlichkeit des linken Warzenfortsatzes, im Zusammenhang nit der anamnestischen Thatsache, dass eine linksseitige Ohreneiterung bestanden hatte, die mit Aufneisselung des Antrum beliandelt worden war, konnte kaum einen Zweifel darüber lassen, dass diese Erkrankung des Gehirns ihren Ausgang vom Ohr genommen hatte. Der langsame Puls und die Blutstaung in der Netzhaut deuteten auf einen raumbeschränkenden Prozess im Schädelinnern hin, und die äbrigen Erscheinungen - Kopfschmerz, motorische Schwäche und Hypästhesie der rechten Seite, Benommenheit, Erbrechen, Sclıwindel, geringe Temperatursteigerungen - passten reeht gut in den Rahmen des Bildes eines Schläfenlappenabscesses, wenn auch die bei linksseitigem Sitz desselben zu erwartende Aphasie fehlte. Gegen eiterige Meningitis sprach hauptsächlich der regelmässige Puls, sowie das Fellen von Nackenstarre und hohem Fieber. Somit hatte, wenngleich eine nachweisbare Eiterung am Ohre nicht bestand, die Diagnose „Schläfenlappenabscess" die meiste Wahrscheinlichkeit für sich; eine sichere Diagnose konnte aber erst aus dem Befunde bei der Operation und ihrem Ergebniss, sowie aus dem damit eingeleiteten Ablauf der Krankheit gestellt werden.

Ein Abscess wurde bei der Operation nicht gefunden; die Aussicht, einen solchen zu finden, wurde gleich bei Beginn der Operation noch unsicherer als vorher dadurch, dass im Warzenfortsatz kein eiteriger, sondern ein nekrotisirender Prozess vorgefunden wurde. Dagegen liess schon die wiederholte Ansaugung von seröser Flüssigkeit bei den Probepunctionen und noch mehr der reichliche Abfluss solcher Flüssigkeit während der Operation nach dem Einschnitt in die harte Hirnhaut eine krankhafte Vermehrung der Gehirnflüssigkeit erkennen, und zur Gewissheit wurde diese krankhafte Vermehrung durch die tagelang andauernde mächtige Durchtränkung unserer dicken Verbände. Diese massenhafte Flüssigkeit konnte nur als das krankhafte Product eines entzündlichen Vorgangs und hier nach Lage der Dinge als das Exsudat der entzündeten weichen Hirnhaut gedeutet werden. Zwar regenerirt sich der normale Liquor cerebrospinalis auch ohne entzündliche Reizung ausserordentlich schnell, so dass z. B. bei Schädelbasisbrüchen grosse Mengen davon abfliessen können; solche Mengen jedoch wie hier dürften bei Schädelbrüchen, sofern nicht auch da eine entzündliche Reizung der Meningen hinzutritt, nicht vorkommen, und jedenfalls haben wir solche Mengen bei unseren zahlreichen sonstigen Gehirnoperationen nie beobachtet. Auf den negativen Ausfall der Trommer'schen Probe mit der ausgelaugten Wundflüssigkeit möchte ich hinsichtlich der Diagnose weniger Werth legen, da in dieser Verdünnung wohl auch der normale Liquor seine an sich schon schwache reducirende Eigenschaft einbüssen dürfte. Aber die Thatsache, dass vor der Operation schwere, das Leben bedrohende Symptome vorhanden waren und dass fast unmittelbar nach der Operation diese bedrohlichen Erscheinungen verschwanden, spricht mit Sicherheit dafür, dass die abfliessende Flüssigkeit es gewesen ist, welche die Raumbeschränkung verursachte; das thut aber nur eine entzündlich vermehrte, niemals die normaler Weise vorhandene Gehirnflüssigkeit. Ueber die Diagnose Meningitis serosa kann daher kein Zweifel bestehen.

Was dann meine Auffassung der Meningitis als einer externa anbelangt, so gründet sich diese darauf, dass die Flüssigkeit unmittelbar unter der Dura hervorquoll und dass eine Eröffnung des Seitenventrikels sicher nicht stattgefunden hat. Es wäre zwar denkbar, dass auch in den Ventrikeln vermelırte Flüssigkeit vorhanden gewesen und diese durch die natürlichen Communications- 
stellen der Ventrikel mit den subarachnoidealen, bezw. subduralen Räumen - Foramen Magendii, Foramina Luschkae - nach aussen abgeflossen wäre; doch führt die Meningitis ventricularis nach Boenninghaus gewöhnlich zu einem activen oder passiven Verschluss jener Stellen. Ausserdem aber spricht einmal das gänzliche Ausbleiben von Hirnprolaps während der Nachbehandlung und dann die Entstehung der Meningitis in unserem Falle dafür, dass sie - in der Hauptsache wenigstens und von vornherein eine externa gewesen ist: sie hat ihren Ausgang genommen von dein krankhaft veränderten Knochen in der Umgebung der alten Antrumwunde, bezw. von einem Reiz, dem die bei der ersten Operation freigelegte Dura in Folge des klankhaften Prozesses in der Wundhöhle, der schliesslich zur Resolption des ganzen Höhleninhalts und hiermit wieder zum völligen Blossliegen jenes früher freigelegten Stückes Dura, sowie zur völligen Nekrose der knöchernen Wandungen der Wundhöhle führte, dauernd ausgesetzt war. Dieser Prozess war nicht eiterig, darum wal auch die von ih inducirte Entzündung der Meningen nicht eiterig; der klankhafte Prozess an den Meningen blieb, da Eitererreger nicht vorhanden waren, gewissermaassen auf der ersten Stufe der eiterigen Meningitis, im Stadium der entzündlichen Hyperämie und seıösen Durchtränkung stehen.

Für den chronischen Charakter der Meningitis schliesslich sprechen die schon seit Jahren bestehenden Kopfschmerzen. ferner das allmähliche Entstehen der Affection von dem in der Antrlumwundhöhle sich ganz allmählich abspielenden nekrotisirenden Prozess aus, und schliesslich die Thatsache, dass durch die Operation nicht alle krankhaften Symptome, insbesondere nicht die Sensibilitäts- und die Geh-, bezw. Gleichgewichtsstörungen beseitigt worden sind; hieraus geht hervor, dass der Prozess an den benachbarten Hirnpartieen bereits dauernde Zerstörungen durch Druck des Exsudats, durch anhaltende seröse Durchtränkung einzelner Hirnabschnitte u. s. w. hervorgerufen haben muss, was doch nur bei längerem Bestehen des Prozesses möglich gewesen sein kann. Auch die in jüngster Zeit wieder beobachteten Beschwerden Kopfschmerzen, Erbrechen - deuten darauf hin, dass der Prozess in chronischer Form auch jetzt noch besteht.

Für die Prognose der Operation ist hieraus zul ersehen, dass bei acutem Charakter des Leidens wohl eine Beseitigung sämmtlicher Symptome und eine endgiltige Heilung durch den Eingriff erwartet werden hann, dass aber bei chronischer Meningitis mit der Wahrscheinlichkeit gerechnet werden muss, dass dauernde Störungen selbst dann, wenn die Operation glücklich und lebensrettend verläuft, zurückbleiben; und selbst quoad vitam ist die Prognose in chronischen Fällen mit Rücksicht auf die möglicherweise eintretenden Rückfälle zum mindesten zweifelhaft.

Die acute Verschlimmerung in unserem Falle kurz vor der Operation ist wohl damit zu erklären, dass von der Meningitis aus plötzlich eine entzündlich-seröse Durchtränkung der Hirnsubstanz linkerseits in der Nähe des Ausgangspunktes der Meningitis, also hauptsächlich am Schläfenlappen stattgefunden hat; anders sind wenigstens die damals zu beobachtenden Heerdsymptome, insbesondere die Ptosis, dann aber auch die motorische Schwäche und die Sensibilitätsherabsetzung auf del lechten Körperhälfte nicht zu erklären, da eine Meningitis serosa allein in Folge der gleichen Druckverhältnisse im ganzen Schädelinnern solche auf bestimmte Organe, bezw. auf eine bestimmte Seite beschränkte Erscheinungen nicht hervorrufen kann. Die exacte Diagnose würde also in unserem Falle zu lauten haben: Meningitis serosa externa chronica und Encephalitis serosa acuta sinistra. Für die Operation ergiebt sich hieraus die Lehre, dass Einschnitte in das Gehirn und nachherige Drainage der Hirnwunde mit Gazestreifen nicht überflüssig sind.

$\mathrm{Ob}$ in unserem Falle die Lumbalpunction ebenso wie unsere Aufmeisselung des Schädels zum Ziele geführt haben wüldde, scheint mir mit Rücksicht auf die überaus grosse Menge des Exsudats, das sich noch eine ganze Reihe von Tagen nach der Operation immer von neuem regenerirte und entleerte, zweifelhaft; zum mindesten hätte die Punction öfter wiederholt werden müssen. Die Radicaloperation am Ohre jedoch wäre zur Beseitigung des Ausgangspunktes der Meningitis und auch zu diagnostischen Zwecken unbedingt erforderlich gewesen: nachdem aber dieser Eingriff gemacht war, konnte auch unbedenklich die viel einfachere und kürzere Trepanation des Schädels in derselben Narkose folgen, zumal ja ein Gehirnabscess trotz des negativen
Ausfalls der Plobepunction noch nicht auszuschliessen war. Ich glaube daher, dass in Fällen wie dem vorliegenden das radicale Vorgehen, die dauernde Drainage des Cavum cranii von aussen her, der blossen Lumbalpunction prinzipiell vorzllziehen ist.

\section{b. Meningitis serosa interna acuta.}

Frau G., 31 Jalıre. Mit 12 Jahren Ohrenlanfen nach Diphtherie 6 Monate lang, seitdem keine Ohreneiterung mehr bemerkt. Mit 27 Jahren einmal Schmerzelı im linken Ohr und Schwerhörigkeit. Sonst nie Ohrenbeschwerden. Rachitisch will sie als Kind nicht gewesen sein; es scheint mir dies ron Interesse, weil damit dieses ätiologische Moment für den Hydrocephalıs internus, der sich anf rachitischer Basis iibrigens viel chronischer und in jugendlicherem Alter als hier z.11 entwickeln pflegt, wegfällt.

Ende September 1898 aus unbekannter Ursache Flimmern vor deıl Alugen; konnte schlecht sehen, nicht lesen, keine Nadel einfädeln. Augenärztliche Behandlung ohne Erfolg. Ende October gesellten sich Kopfschmerzen, Schwindel, Schlaflosigkeit hinzı, ferner Erbrechen, das täorlich mehrmals anftrat und die Kranke anch anf der Strasse wiederholt iiberraschte. Zunehmende Schwäche, vom 16. November an musste sie deshalb das Bett hïten.

21. November, Aufnahme in die Charité, zınächst Nervenabtheilung. Hier dachte man zinüchst an einen Tumor cerebri; da bemerkte man am 28. November, dass das linke Ohr eiterte; die Kranke wurde zur Untersuchung anf die Ohrenstation gebracht ınd am 2. December behufs Operation zil uns verlegt.

Klagen: Grosse Schwäche, kann nur einige Schritte allein gehen; Schwindel und Kopfschmerzen, beides soll die Tage vorher noch stärker gewesen sein; Erbrechen, Gedächtnissschwäche.

Statıs: Dïrftig genährte Frau. Innere Organe gesund; keine Tuberknlose. Harn frei von Eiweiss und Zucker. Stıhl nicht angehalten. Gang unsicher; bei Romberg starkes Schwanken. Patellarreflex normal. Kein Fieber. Pıls wechselnd, 66, 72, 80, in sich regelmässig, nicht auffallend gespannt. Keinerlei Anzeichen von ïberstandener oder bestehender Liles, die anch anamnestisch bestimmt in $\mathrm{Ab}$ rede gestellt wird; die Kranke hat drei gesinde Kinder. Für überstandene Rachitis spricht ebenfalls nichts.

Sensorium nicht ganz frei, giebt öfter falsche, verwirrte Antworten, das Gedächtniss hat für die juingsten Vorgänge stark gelitten, die genane Anamnese ist daher erst später alfgenommen worden. Lähmungen, Aphasie, Ataxie fehlen. - Schläfengegend links percussionsempfindlich.

Pupillen weit, reagieren nicht. Augenhintergrund rechts blassroth, ödematös, Arterien nicht zul sehen, Venen prall gefüllt; die geröthete Papille sticht nicht vom übrigen Fundus ab. Links derselbe Befınd, doch nicht ganz so intensiv, Papille noch abgrenzbar; oben einige Hämorrhagieen. Angenbewegongen frei, kein Nystagmus (St.-A. Br e cht).

Ohren: Rechts kleine Verkalkung im Trommelfell, sonst normal. Links, äusserer Gehörgang mit stinkendem Eiter verschmiert, ziemlich eng; Trommelfell geröthet, verdickt, hoher Defekt über dem kurzen Fortsatz. Fliistersprache rechts normal, links $10 \mathrm{~cm}$. Warzenfortsatz beiderseits etwas druckempfindlich, links mehr als rechts.

Die allgemeinen Beschwerden - benommenes Sensorium, Kopfschmerz, Schwindel, auch das Erbrechen - deuteten anf eine cerebrale Affectioll, der Augenbefund wies auf eine Raumbeschränkung im Schädelinnern hiı, wenn auch ausgesprochene Pulsverlangsamung nicht bestand. Dass das Erbrechen und der Schwindel besonders in einer bestimmten Seitenlage aufgetreten wäre, wurde nicht beobachtet; damit war die Anwesenheit eines verschieblichen Tumors ausgeschlossen. Die Ohreneiterung machte es dagegen wahrscheinlich, dass die Affection otitischen Ulsprnngs sei, und Mangels aller weiteren Hinweise wurde wegen der Percussionsempfindlicllkeit der linken Schläfengegend in erster Linie an einen linksseitigen Schläfenlappenabscess gedacht. Herr Geh. Rath Trantmann schritt daher zur Operation am

3. December 1898. Radicaloperation links und Trepanation auf den linken Schläfenlappen. Es fand sich ein Cholesteatom des Antrum, Aditıs und Recessus epitympanicus. -- Trepanation von allssen in derselben Weise wie bei Fall a, nur dass hier die Kreissäge benutzt wurde. Dura pulsirte nicht, stülpte sich aber in der Knochenwunde prall nach aussen vor. Dura krenzförmig gespalten, Einschnitte in den Schläfenlappen nach mehreren Richtıngen hin, $3 \mathrm{~cm}$ weit, Erweiterung mit der Kornzange, kein Eiter. 4. December Befund im wesentlichen wie vor der Operation, nul dass - trotz der Narkose kein Erbrechen mehr allfgetreten ist. Flüstersprache rechts normal, trotz der tiefen Einschnitte in den linken Schläfenlappen. 6. December Puls 66, trotzdem die Temperatur Morgens auf $37,8^{\circ}$ steigt. Beiderseits noch exquisite Stanungspapille. Schwindel angeblich stärker. Bei forcirtem Blick nach rechts Nystagmus.

Trepanation auf das Kleinhirn links. Hantschnitt nach oben convex iiber einer Linie, die von der Mitte des hinteren Randes der Mastoidwunde zur Protuberantia occipitalis externa gezogen wird. Hantlappen nach minten geklappt, in der Mitte der angegebenen Linie wird ein Knochenrechteck mit Kreissäge nind Meissel herausgenommen, $3 \mathrm{~cm}$ 
lang. $1 \frac{1}{2} \mathrm{~cm}$ breit. derart. dass die obere Längskante des Rechtecks in der mehrgenannten Linie liegt. Einschnitt in die Dura und dann in das Kleinhirn. $3 \mathrm{~cm}$ tief. Erweiterung mit der Kornzange; kein Eiter.

Im Gegensatz zu Fall a trat hier auch nach dieser Operation keine auffallende unmittelbare Besserung ein.

Am 8. December beim Verbandwechsel keine auffallende Durchtränkung der Verbandstücke: an der Schläfenwunde mässiger Hirnprolaps. oberflächlichste Schicht davon nekrotisch. - - 11. December. Stauungspapille hat zugenommen: Netzhaut $+2 \mathrm{D}$. Papille $+6-7 \mathrm{D}$. Subjectives Befinden etwas besser: hat leidlich geschlafen.

16. December. Stauungspapille hat noch mehr zugenommen (9 D). in der Netzhaut sind frische Blutungen aufgetreten (St.-A. Brecht).

Ganz allmählich entwickelte sich jetzt an beiden Hirnwunden ein ganz mächtiger Prolaps: die obersten Schichten waren bei jedem Verbandwechsel nekrotisch und wurden, soweit sie sich nicht von selbst abstiessen, abgetragen.

Von Mitte December an 0.1 Calomel täglich. Gegen Ende December hin verschlechterte sich das Allgemeinbefinden: öfter Abends $38.0^{\circ}$. Benommenheit. bisweilen wieder Erbrechen. Keine Aplasie. aber bei den Worten werden einzelne Buchstaben verwechselt (Merser statt Müller. Märker oder Gerrier statt Gärtner), auch ganze Begriffe werden verwechselt. z. B. sagt sie ...an- und ausziehen“ statt ..den Ver" band wechseln“.

2. Januar. Hirnprolaps an beiden Wunden noch gewachsen. Stauungspapille geringer (7.5 D. Retina 2 D). keine neuen Blutungen. - Leichte Gingivitis. Calomel ausgesetzt. Jodkali.

Um diese Zeit war der Höhepunkt der Krankheit erreicht: von jetzt ab trat. Anfangs ganz unmerklich. später augenfälliger. Besserung ein. Zuerst besserte sich das subjective Befinden. während der Hirnprolaps. wie am 5., 7. und 9. Januar bei jedem Verbandwechsel konstatirt werden musste, noch weiter beträchtlich zunahm, wobei die oberflächlichen Partieen sich fortgesetzt reichlich abstiessen: erst am 21. Januar war hieı der Höhepunkt erreicht. der Prolaps war am Schläfenlappen fast hühnereigross.

13. Januar. Verbandstïcke heute auffallend stark serös durch feuchtet; nach ihrer Wegnahme entleert sich aus der Temporalwunde reichlich seröse Flüssigkeit.

15. Januar. Verbandstücke wieder stark durchfeuchtet. viel stärlker als bei den frïheren Verbänden.

16. Januar: Augenbefund: Schwellung der Papillen nur noch gering. Grenzen aber stark verwaschen. Venen stark gefüllt und geschlängelt. - Kein Kopfschmerz. Schlaf gut.

18. Januar. Fühlt sich völlig wohl und äussert den Wunsch. aufzustehen. was aber noch nicht gestattet wird. Puls 70-80. Verbandstücke nicht mehr aussergewöhnlich feucht. - Jodkali ausgesetzt.

25. Januar. Vom Hirnprolaps stösst sich nichts mehr ab. die Oberfläche sieht frischroth aus. Keine Beschwerden. Täglich eine Stunde im Lelınstuhl. Stuhlgang ohne künstliche Nachhilfe.

30. Januar. Hirnprolaps an beiden Wiunden deutlich kleiner geworden. Mehrere Stunden ausser Bett. Geht einige Schritte. ohne zu schwanken. Stauungspapille noch vorhanden, aber Schwellung deutlich abgenommen.

Die prolabirte Hirnmasse wurde allmählich von allen Seiten durch dicke Graululationen überwuchert. unter deren bindegewebiger Umwandlung und narbiger Schrumpfung sich der Prolaps verkleinerte. und zwar ging dies Anfang Februar ganz überraschend schuell, fast zusehends vor sich. Selbstverständlich ist hierbei der Umstand förderlich gewesen. dass gleichzeitig das Exsudat in den Ventrikeln allmählich resorbirt wurde und damit der Druck von. innen her nachliess. Am 7. Februar bereits war die Kleinhirnwunde vernarbt. die Schläfenwunde am 27. Februar. Heilungsdauer also für die Kleinhirıwunde zwei Mo-

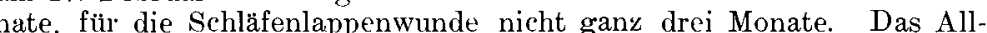
remeinbefinden liess nichts zu wünschen übrig: die Kranke half von Mitte Februar an bereits kleine Handleistungen verrichten. Beim Bücken nocls etwas Schwindel. sie geht daher. wenn sie etwas aufheben will. lieber in die Kniebeuge, anstatt sich zu bücken: im ganzen ist sie noch ziemlich schwach. Körpergewicht war auf 91 Pfund heruntergegangen. beträgt Anfang März wieder 97 Pfund.

14. März. Radicaloperationswunde an der hinteren Wand noch epidermisfrei. Keine Beschwerden mehr. Augenbefund: Papillen beiderseits noch geröthet. Grenzen unscharf: Refraction der Retina $2 \mathrm{DH}$. des Opticus $3 \mathrm{DH}$. also noch Papillenschwellung von $1 \mathrm{D}(=1 / \mathrm{mm})$ Keine Hämorrhagien mehr. L. $\$ 5 / 15 . \quad$ R. $\$ 5 / 20.0 .5$ in $16 \mathrm{~cm}$. Gläser bessern nicht (St.-A. Brecht)

Am 15. März wurde die Frau als gebessert auf ihren Wunsch entlassen. Sie steht seitdem ihrer Wirthschaft - Mann und drei Kinder - wieder selbständig und allein vor und kommt zum Verbandwechsel an der Radicaloperationswunde alle zwei Tage allein zur Charité. Am 12. A pril war auch diese Wunde völlig geheilt. Heilungsdauer $4 \frac{1}{8}$ Monat. Flüstersprache $1.0 .5 \mathrm{~m}$. Irgendwelche krankhaften Erscheinungen. wie Störungen der Sensibilität. der Motilität. der Sprache u. s. w.. sind nicht zurückgeblieben. auch die Kräfte haben sich allmählich gelıoben. so dass hier von einer vollständigen Heilung gesprochen werden kaın.
Wie im Falle a so ist auch hier die Diagnose erst dưrch den Verlauf des Krankheitsprozesses ermöglicht worden. Aber im Gegensatz zu jenem Falle war hier die Diagnose noch eine Reihe von Wochen nach den operativen Eingriffen zweifelhaft. während dort das unmittelbare Ergebniss der Operation die Diagnose alsbald sicherte.

Dass kein Gehirnabscess vorlag. das ergab auch im Falle $b$ unmittelbar die Operation am 3.. bezw. 6. December: da aber nach der doppelten Eröffnung der Schädelkapsel die krankhaften Erscheinungen unverändert fortbestanden. so war über deren Ursache trotz der Operationen noch keine Klarheit geschaffen. Zunächst kehrten wir zu der ursprünglich gestellten Diagnose ..Tumor cerebri" zurück und gaben im Hinblick auf die Möglichkeit. dass es sich um ein Gummi handeln könnte. erst Calomel. dann Jodkali. Triftige Gründe veranlassten uns aber im weiteren Verlauf. diese Diagnose wieder fallen $\mathrm{zu}$ lassen. Als nämlich mit der eintretenden Besserung die Frau im Stande war, uns anamnestische Angaben zu machen. bestritt sie eine luetische Infection auf das bestimmteste; es waren auch objectiv an ihr keinerlei Anzeichen von Lues zu entdecken; ihre Kinder sind alle vollständig gesund. Ausserdem nahm der Hirnprolaps auch nach dem Aussetzen des Jodkali noch zu. das Gummi wäre also durch das Calomel und das Jodkali gänzlich unbeeinflusst geblieben. Es musste also dem erhöhten Druck im Schädelinnern eine andere Ursache zu Grunde liegen. und das konnte nach Ausschluss eines $\Lambda$ bscesses und eines Tumors sowie einer Meningitis externa nur eine Meningitis interna mit starkem Exsudat in den Ventrikeln sein. Eine Punction des linken Seitenventrikels von der Schläfenwunde oder des vierten Ventrikels von der Kleinhirnwunde her wäre geeignet gewesen, die Diagnose zur Evidenz zu sichern; leider ist die Punction unterblieben. und zwar aus verschiedenen Gründen. Einmal rechneten wir anfangs noch nicht mit der Möglichkeit eines serösen Ergusses in die Ventrikel. und als daran gedacht werden musste. hatte sich der Zustand durch das Ausweichen des Gehirns aus den Trepanationsöffnungen heraus soweit gebessert. dass die Punction eben nur noch einen diagnostischen. aber keinen therapeutischen Werth mehr gehabt hätte. Da aber um diese Zeit die Diagnose auch ohne die Punction schon genügend gesichert erschien. wurde von dem Eingriff endgiltig Abstand genommen.

Ihren das Leben bedrohenden Charakter verloren die von dem Exsudat ausgehenden Hirndruckerscheinungen mit dem Augenblick. in welchem durch Eröffnung der Schädelkapsel dem Gehirn die Möglichkeit. auszuweichen. sich auszudehnen. gegeben war; so konnte unter stetigem Ausweichen der Hirnmasse und unter stetiger Zunahme des Prolapses der entzündliche Prozess und das Exsudat in den Ventrikeln ohne Lebensgefahr für die Kranke seinen Höhepunkt erreichen. worauf dann das Exsudat nach dem Aufhören des entzündlichen Prozesses. dessen Ausgangspunkt mit der Radicaloperation am Ohre ja entfernt war. zur Resorption kam; diese Aufsaugung dürfte mit Rücksicht auf die schnelle Wundheilung und die rasche Besserung im Befinden der Kranken nach dem Ueberschreiten des Höhepunkts ziemlich schnell vor sich gegangen sein.

Zweifellos würde eine Ventrikelpunction entweder gleich bei der Eröffnung des Schädels oder doch um die Zeit. als der Hirnprolaps deutlich in die Erscheinung trat. eine Besserung des subjectiven Befindens und wahrscheinlich auch eine Abkürzung des Krankheitsverlaufs herbeigeführt haben. und unser Fall lehrt daher dass man bei ähnlicher Sachlage, wenn nach einer Trepanation der vermuthete Abscess nicht gefunden worden ist und die schweren Erscheinungen unveründert fortbestehen. auch an die Möglichkeit einer Meningitis ventricularis denken und therapeutisch rechtzeitig demgemäss verfahren soll.

Bemerkenswerth ist an unserem Falle ferner. welch grosse Mengen von Gehirnsubstanz verloren gehen können. ohne dass der Kranke einen ersichtlichen Schaden dadurch erleidet Die Hirnsubstanz zwischen Ventrikel und Schläfenwunde hatte sich durch oberflächliche Abstossung. bezw. Abtragung allmählich so sehr verdünnt. dass in der Zeit zwischen 13. und 15. Januar - als weiterer Beweis für unsere Diagnose - die Ventrikelflüssigkeit. entweder durch die dünne Schicht der noch verbliebenen Hirnmasse hindurch diffundirend oder vielleicht auch durch einen in Folge des inneren Druckes entstandenen Spalt sich ergiessend. in die Verbandstücke austreten konnte. In der That sind in unserem Falle ganz überraschend grosse Mengen Hirn- 
masse vom Schläfenlappen, aber auch vom Kleinhirn verloren gegangen, und doch ist wunderbarer Weise nicht die Spur irgend welcher Ausfallserscheinung danach zurückgeblieben.

Der starke Prolaps giebt mir schliesslich noch zul einer Bemerkung Veranlassung: hätten wir, wie mehrfach vorgeschlagen worden ist ${ }^{1}$ ), den vermutheten Hirnabscess von der Radicaloperationswundhöhle aus zı öffnen gesucht, so würde das prolabirende Gehirn vom Schläfenlappen und vom Kleinhirn her in diese Knochenhöhle hineingetreten sein. Das würde zwei Nachtheile, bezw. Gefahren mit sich gebracht haben: Ein regelrechtes Verbinden der Wundhöhle, namentlich ein Austamponiren ihrer Tiefe, wäre unmöglich geworden ${ }^{2}$ ), und es ist höchst wahrscheinlich, dass es von dem in der Tiefe der Wunde verhaltenen und sich schliesslich zersetzenden Wundsecret sowie von den sich dort abstossenden, aber nicht genïgend entfernbaren und daher zerfallenden Gehirnmassen aus schliesslich doch zu einer tödtlichen eitrigen Meningitis gekommen wäre. Und der zweite Nachtheil wäre der gewesen: Bei dem engen Raum in der Knochenwundhöhle hätte möglicher Weise das Gehirn nicht genügend Raum zum Ausweichen gefunden, und es wäre dann trotz unserer Operationen, auch ohne den Hinzutritt einer tödtlichen Meningitis purulenta, in Folge des Hirndruckes zum tödtlichen Ausgang gekommen. Es empfiehlt sich daher - ganz abgesehen von allen anderen Gründen - stets, so wie es auf der Trautmann'schen Klinik der Charité auch grundsätzlich geschieht, Gehirnabscesse nicht von der Ohrenoperationswunde, sondern von aussen her in Angriff zu nehmen, da man auch in den Fällen, in denen man einen Hirnabscess wirklich findet, vor einem nachfolgenden stärkeren Hirnprolaps nie sicher ist. 